\title{
Polaprezinc reduces the severity of radiation-induced mucositis in head and neck cancer patients
}

\author{
HIROSHI DOI ${ }^{1}$, MASAYUKI FUJIWARA ${ }^{1}$, HITOMI SUZUKI $^{1}$, YASUE NIWA ${ }^{1}$, MASAHIRO NAKAYAMA ${ }^{2}$, \\ TOSHIYUKI SHIKATA ${ }^{2}$, SOICHI ODAWARA ${ }^{1}$, YASUHIRO TAKADA $^{1}$, TAKESHI KIMURA ${ }^{2}$, \\ NORIHIKO KAMIKONYA $^{1}$ and SHOZO HIROTA $^{1}$ \\ ${ }^{1}$ Department of Radiology, Hyogo College of Medicine; ${ }^{2}$ Department of Pharmacy, \\ The Hospital of Hyogo College of Medicine, Nishinomiya, Hyogo 663-8501, Japan
}

Received September 16, 2014; Accepted December 12, 2014

DOI: $10.3892 / \mathrm{mco} .2014 .479$

\begin{abstract}
Polaprezinc (PZ), an antiulcer drug, has been reported to have antioxidant properties. The aim of the present study was to assess the feasibility and efficacy of administering PZ for radiation-induced mucositis in head and neck cancer patients. Patients with newly diagnosed head and neck cancer were enrolled in this prospective study. PZ was prepared as an oral rinse. The $\mathrm{PZ}$ oral rinse was used four times per day during the course of radiotherapy. Sequential changes in radiation mucositis were assessed during and after radiotherapy according to the Common Terminology Criteria for Adverse Events, version 3.0. Furthermore, a retrospective comparison analysis was performed to assess the efficacy of PZ for radiation-induced mucositis. A total of 32 patients were enrolled in the prospective study of the PZ oral rinse. Radiotherapy was performed up to a total dose of 60-66 Gy using a conventional schedule combined with chemotherapy. Of the 32 patients, 30 (93.8\%) reported no complaints due to the PZ oral rinse. In addition, PZ was not associated with severe adverse effects. Among the patients who received PZ, grade 3 mucositis was observed in $29.0 \%$ based on the mucosal findings and in $39.3 \%$ based on the symptoms. In the patients who did not receive PZ, the incidence of grade 3 mucositis was $40.0 \%$ based on the mucosal findings and $60.7 \%$ based on the symptoms. Moreover, PZ promoted the recovery from mucositis caused by chemoradiotherapy and was not associated with reduced tumor response to radiotherapy. Therefore, the PZ oral rinse was well tolerated and proved to be efficient for the treatment of radiotherapy-induced oral mucositis.
\end{abstract}

Correspondence to: Dr Hiroshi Doi, Department of Radiology, Hyogo College of Medicine, 1-1 Mukogawa-cho, Nishinomiya, Hyogo 663-8501, Japan

E-mail: h-doi@hyo-med.ac.jp

Key words: polaprezinc, mucositis, head and neck cancer, oral rinse, radiotherapy

\section{Introduction}

Radiotherapy is crucial for the management of head and neck cancer, either as definitive or post-surgical adjuvant treatment (1). Radiation-induced mucositis is the most common toxicity, which often interrupts the planned course of treatment and significantly reduces a patient's quality of life (QOL) during the treatment (2). Amifostine has been reported to prevent severe radiation-induced toxicities in head and neck cancer patients and is the only radioprotectant that has been approved by the US Food and Drug Administration (3). However, the daily use of amifostine is limited due to its hematological and gastrointestinal toxicity. Therefore, there is no standardized treatment for chemoradiotherapy-induced mucositis.

Polaprezinc (PZ; Zeria Pharmaceutical Co., Ltd., Tokyo, Japan) is an antiulcer drug and chelate compound consisting of a zinc ion, L-carnosine, a $\beta$-alanine dipeptide and L-histidine. It has been reported that PZ has antioxidant properties and scavenges free radicals (4-7). We previously reported the efficacy of PZ for acute radiation proctitis in an animal model and demonstrated that PZ exerts anti-inflammatory effects following radiation exposure (8). Watanabe et al (9) recently reported that $\mathrm{PZ}$ is effective for mucositis induced by chemoradiotherapy in head and neck cancer patients. Swallowing is often difficult for patients with mucositis, including an oral rinse. In addition, the direct effects of $\mathrm{PZ}$ on the mucosa remain unclear, although PZ is likely to exert direct anti-inflammatory effects on the damaged mucosa (8). Therefore, we prepared a $\mathrm{PZ}$ oral rinse using a new protocol, which yields a rinse with a good stability and a stronger adhesion activity, to enhance the direct effects of PZ on the oral and oropharyngeal mucosa (9).

In the present study, we investigated the feasibility of the newly designed $\mathrm{PZ}$ oral rinse in head and neck cancer patients in a prospective clinical trial and also assessed the efficacy of PZ for radiation-induced mucositis in a case-matched study with a larger number of patients.

\section{Materials and methods}

Patients. A total of 32 patients with newly diagnosed head and neck cancer who underwent radiotherapy at the Hospital of Hyogo College of Medicine between March, 2010 and 
February, 2013 were enrolled in this prospective study [PZ (+) group]. Adult patients with pathologically confirmed head and neck cancer, who were scheduled to receive radical radiotherapy and who provided written informed consent, were considered eligible for participation in this study. Patients who received postoperative radiotherapy for head and neck cancer were excluded. This study was approved by the Ethics Committee of Hyogo College of Medicine (no. 791).

Preparation of the $P Z$ oral rinse and treatment schedule. The preparation of and treatment with the $\mathrm{PZ}$ oral rinse was previously described in detail (10). In brief, PZ was specifically prepared for the present study as an oral rinse using carboxyvinyl polymer (HIVISWAKO105; Wako Pure Chemical Industries, Ltd., Osaka, Japan) as a base. The $\mathrm{pH}$ was adjusted to 7.0 with sodium hydrogen carbonate (Yoshida Pharmaceutical Co., Ltd., Tokyo, Japan). PZ was suspended to provide a final concentration of $37.5 \mathrm{mg} / 10 \mathrm{ml}$, according to the standard dose of PZ for the treatment of gastric ulcer (150 mg/day). We confirmed the stability of the PZ oral rinse prior to the treatment of patients as previously described (10). A 10-ml aliquot of the PZ oral rinse was used four times a day. The oral rinse was retained for $1 \mathrm{~min}$ and was then spat out without swallowing. No ingestion of food or drink was permitted for $30 \mathrm{~min}$ following treatment. The treatment with the PZ oral rinse was repeated during the entire course of radiotherapy. A mouthwash with sodium gualenate hydrate was also concomitantly used, but no other mucosal protective agents were permitted during this prospective study. Toxicity was assessed according to the Common Terminology Criteria for Adverse Events, version 3.0 (11).

Radiotherapy. All the patients were placed in the supine position and scanned using an Aquilion LB computed tomography (CT) unit (Toshiba, Ohtawara, Japan). The CT dataset was transferred to the $\mathrm{XiO}$ treatment planning system (Elekta, Stockholm, Sweden), to outline the volumes of interest. Radiotherapy was performed using a three-dimensional conformal radiotherapy technique, which was typically performed using 4-MV photons. The planned radiotherapy was delivered with a dose of 200 cGy once daily using a linac machine (Primus MD2; Siemens, Munich, Germany). Patients with oral cancer received a total prescription dose of $60 \mathrm{~Gy}$ combined with concurrent arterial infusion chemotherapy. Patients with laryngeal and pharyngeal cancer received a total prescription dose of $66 \mathrm{~Gy}$ combined with concurrent chemotherapy.

Case-matched analysis. To evaluate the efficacy of the PZ oral rinse, a case-matched cohort analysis was performed in the present study. Thirty patients with head and neck cancer who underwent radiotherapy at the Hospital of Hyogo College of Medicine between January, 2009 and January, 2010 were retrospectively reviewed based on their medical records and analyzed [PZ (-) group] and compared to the present cohort. The patients in the PZ (-) group underwent radiotherapy using the same protocol as that used in the prospective study of the $\mathrm{PZ}$ oral rinse. A mouthwash with sodium gualenate hydrate was used as supportive treatment, but the patients in the PZ (-) group did not receive PZ. One patient from the prospective cohort who discontinued the PZ oral rinse on the third day
Table I. Characteristics of the patients in the prospective study.

\begin{tabular}{lr}
\hline Characteristics & $\begin{array}{r}\text { No. } \\
(\mathrm{n}=32\end{array}$ \\
\hline Age, years & \\
Median & 67 \\
Range & $41-86$ \\
Gender & \\
Male & 29 \\
Female & 3
\end{tabular}

$\begin{array}{lr}\text { Tumor location } & 6 \\ \text { Oral cavity } & 9 \\ \text { Nasopharynx } & 5 \\ \text { Oropharynx } & 5 \\ \text { Hypopharynx } & 7 \\ \text { Larynx } & \\ \text { Clinical stage } & 6 \\ \text { I } & 7 \\ \text { II } & 7 \\ \text { III } & 11 \\ \text { IVA } & 1 \\ \text { IVC } & \end{array}$

Pathological type

Squamous cell carcinoma $\quad 31$

Lymphoepithelial carcinoma $\quad 1$

Total radiotherapy dose, Gy

66

24

62

60

1

7

Chemotherapy

CBDCA $\quad 8$

TXT 18

$\begin{array}{ll}\mathrm{CDDP}^{\mathrm{a}} & 6\end{array}$

${ }^{\mathrm{a}}$ Cisplatin was delivered using an arterial infusion technique. CBDCA, carboplatin; TXT, docetaxel; CDDP, cisplatin.

and dropped out of the chemoradiotherapy protocol due to a severe gastric ulcer that developed during the fifth week was excluded from the comparative analysis.

Statistical analysis. The data are expressed as medians and range, unless otherwise indicated. A statistical analysis was performed to compare the two groups. Parametric data were analyzed using a t-test and non-parametric variables were analyzed by a two-tailed Fisher's test. The Chi-square test was first used to assess the trend in more than two groups. The Fisher's exact test was then used to assess the significance of the differences between each group and the other groups. The outcomes were estimated with Kaplan-Meier statistics and compared using the log-rank test. The Prism 6.0b software (Graph Pad Software, Inc., La Jolla, CA, USA) was used for the statistical analysis. $\mathrm{P}<0.05$ was considered to indicate a statistically significant difference. 
Table II. Characteristics of the patients in the case-matched analysis.

\begin{tabular}{|c|c|c|c|}
\hline Characteristics & $\begin{array}{l}\mathrm{PZ}(+) \\
(\mathrm{n}=31)\end{array}$ & $\begin{array}{l}\mathrm{PZ}(-) \\
(\mathrm{n}=30)\end{array}$ & P-value \\
\hline Age, years & & & 0.87 \\
\hline Median & 67 & 67 & \\
\hline Range & $41-86$ & $21-80$ & \\
\hline Gender & & & 0.47 \\
\hline Male & 28 & 25 & \\
\hline Female & 3 & 5 & \\
\hline Follow-up term, months & & & 0.41 \\
\hline Mean & 25 & 24 & \\
\hline Range & $0-46$ & $0-60$ & \\
\hline Tumor location & & & 0.14 \\
\hline Oral cavity & 6 & 6 & \\
\hline Nasopharynx & 9 & 2 & \\
\hline Oropharynx & 5 & 4 & \\
\hline Hypopharynx & 5 & 10 & \\
\hline Larynx & 6 & 7 & \\
\hline Primary unknown & 0 & 1 & \\
\hline Clinical stage & & & 0.20 \\
\hline I & 5 & 2 & \\
\hline II & 7 & 5 & \\
\hline III & 7 & 8 & \\
\hline IVA & 11 & 14 & \\
\hline IVC & 1 & 1 & \\
\hline Pathological type & & & 0.35 \\
\hline Squamous cell carcinoma & 30 & 27 & \\
\hline Others $^{\mathrm{a}}$ & 1 & 3 & \\
\hline Total radiotherapy dose, Gy & & & 0.17 \\
\hline 66 & 24 & 18 & \\
\hline 60 & 7 & 12 & \\
\hline Chemotherapy & & & 0.0057 \\
\hline CBDCA & 8 & 2 & 0.081 \\
\hline TXT & 17 & 16 & 1.00 \\
\hline $\mathrm{CDDP}^{\mathrm{b}}$ & 6 & 7 & 0.76 \\
\hline $\mathrm{CDDP}+5 \mathrm{FU}$ & 0 & 2 & 0.24 \\
\hline None & 0 & 3 & 0.11 \\
\hline
\end{tabular}

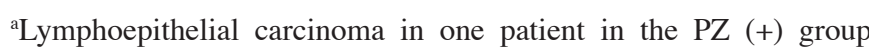
and non-keratinizing carcinoma, papillary cystadenocarcinoma and small-cell carcinoma in one patient each in the PZ (-) group. ${ }^{\mathrm{b} C i s p l a t i n}$ was delivered using an arterial infusion technique. PZ, polaprezinc; CBDCA, carboplatin; TXT, docetaxel; CDDP, cisplatin; 5FU, 5-fluorouracil.

\section{Results}

Feasibility of the PZ oral rinse. A total of 32 patients, including 29 men and 3 women, were enrolled in this prospective study. The median patient age was 67 years (range, 41-86 years). The patient characteristics are listed in Table I. One patient
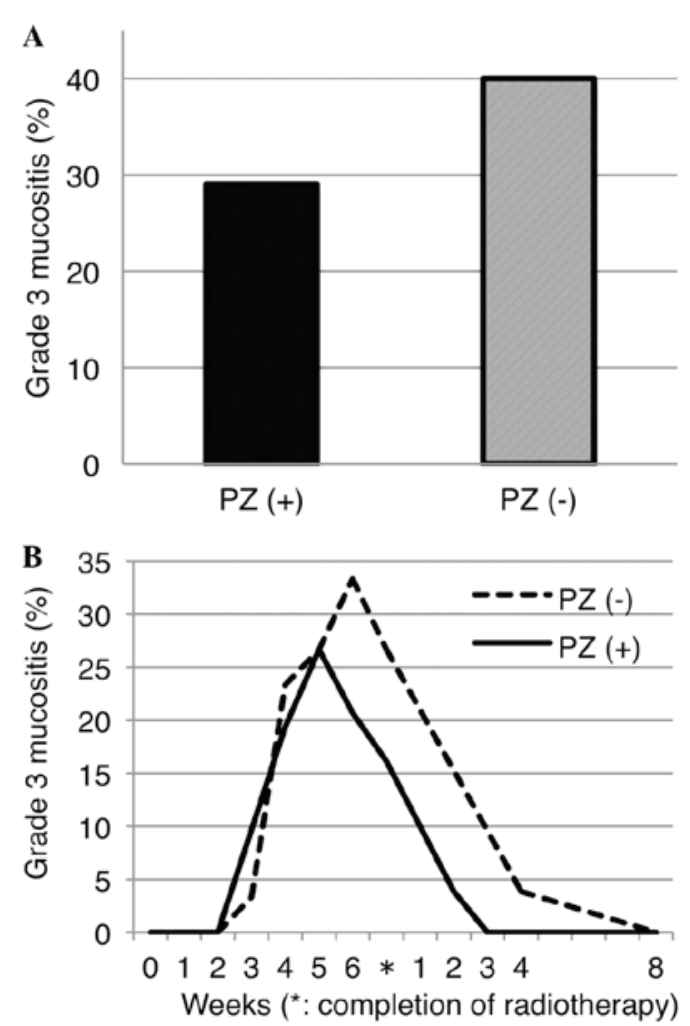

Figure 1. Polaprezinc (PZ) reduced the incidence of severe radiadion-induced mucositis, as indicated by clinical examinations, according to the Common Terminology Criteria for Adverse Events, version 3. Acute radiation mucositis developed in all the patients, as demonstrated by clinical examination in both groups by the fifth week or later after the initiation of radiotherapy. (A) The incidence of grade 3 mucositis was 29.0 and $40.0 \%$ in the PZ (+) and the PZ (-) groups, respectively $(\mathrm{P}=0.42)$. The $\mathrm{PZ}$ treatment ameliorated the mucosal lesions, as indicated by the macroscopic findings. (B) Sequential findings of radiation mucositis during and after radiotherapy. The patients who received $\mathrm{PZ}$ oral rinse recovered from the radiation-induced mucositis within a shorter time period compared to those who did not.

reported discomfort in the pharynx on the third day after treatment initiation. One patient developed nausea on the 22nd day and complained of the same symptom when sodium gualenate hydrate was used as a mouthwash without PZ. Both patients discontinued the PZ oral rinse. The remaining 30 patients (93.8\%) reported no complaints due to the $\mathrm{PZ}$ oral rinse and continued using the $\mathrm{PZ}$ oral rinse during the entire course of radiotherapy. No elevated transaminase levels were observed upon completion of the radiotherapy course.

Efficacy of PZ for radiation mucositis in head and neck cancer patients. The patient characteristics of the PZ (+) and PZ (-) groups are listed in Table II. There were no significant differences in gender, age, tumor location or total dose of radiotherapy between the two groups. The sequential changes due to acute radiation mucositis during the course of radiotherapy are shown in Figs. 1 and 2. The PZ oral rinse significantly reduced the incidence of symptoms and the macroscopic findings and promoted recovery from radiation-induced mucosal damage, although no significant differences were observed between the groups.

PZ does not affect survival outcome. PZ has been reported to reduce the reactive oxygen species in the damaged mucosa (6). 

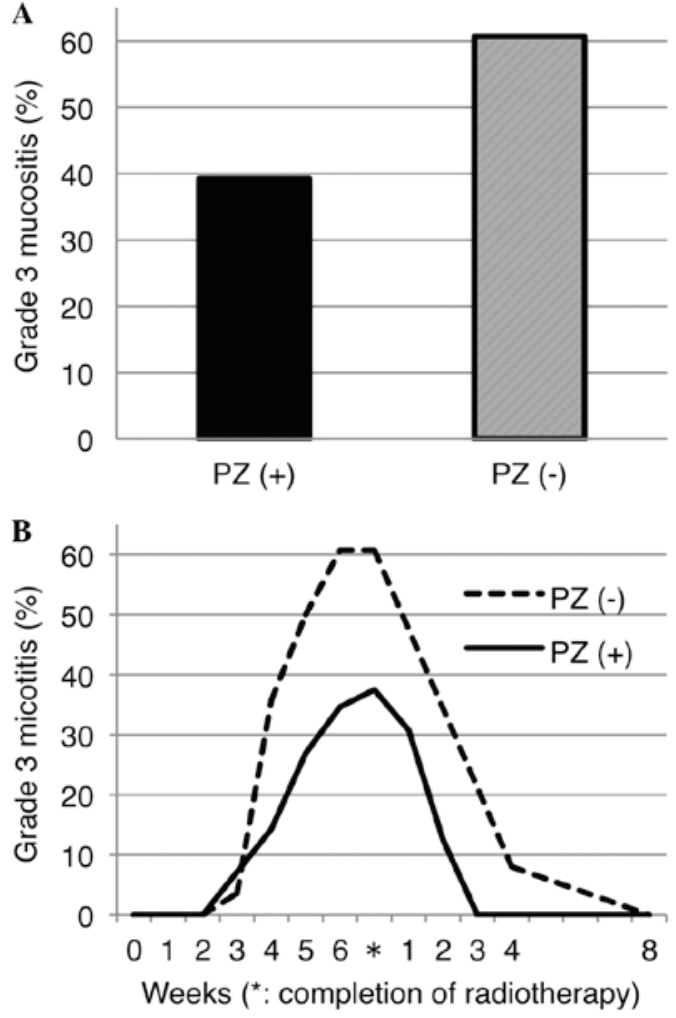

Figure 2. Polaprezinc (PZ) reduced the incidence of severe radiadion-induced mucositis as indicated by the improved ability to eat, according to the Common Terminology Criteria for Adverse Events, version 3. Symptomatic/functional mucositis developed in all the patients, apart from one patient in the PZ $(+)$ group, by the fourth week and gradually worsened until the end of the radiotherapy course. (A) The incidence of grade 3 symptoms was 39.3 and $60.7 \%$ in the PZ (+) and the PZ (-) groups, respectively $(\mathrm{P}=0.18)$. (B) Sequential findings of radiation mucositis during and after radiotherapy. The patients who received $\mathrm{PZ}$ recovered from acute radiation-induced mucositis within a shorter time period compared to those who did not. The data revealed that PZ treatment improved the patient's oral ingestion ability and quality of life.

The negative effects of PZ on cancer therapy have been less extensively investigated, although the use of a radioscavenger may reduce the efficacy of radiotherapy in patient with malignant diseases. To address this issue, we analyzed and compared the survival outcomes of patients treated with and without the $\mathrm{PZ}$ oral rinse. The overall patient survival is presented in Fig. 3 and the survival, disease-specific survival and disease-free survival rates at 2 years after treatment are shown in Fig. 4. There were no significant differences in overall survival, 2-year survival, 2-year disease-specific survival or disease-free survival rates during follow-up between the two groups. However, the PZ (+) group exhibited a tendency for better survival outcomes. In addition, the patients with a follow-up period of at least 1 year were analyzed in terms of late toxicity. The incidence of osteonecrosis of the jaw was 7.7 and $8.0 \%$ in the 26 patients in the PZ (+) group and in the 25 patients in the $\mathrm{PZ}(-)$ group, respectively $(\mathrm{P}=0.97)$ (data not shown).

\section{Discussion}

Although radiotherapy is highly effective for head and neck cancer, painful oral mucositis frequently develops, which

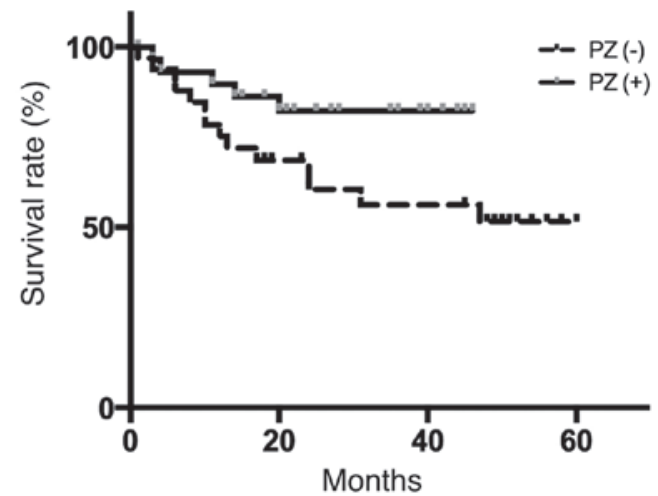

Figure 3. Overall survival of the (PZ) (+) and PZ (-) groups. The patients treated with the $\mathrm{PZ}$ oral rinse exhibited a better survival compared to those who were not $(\mathrm{P}=0.15)$. $\mathrm{PZ}$, polaprezinc.
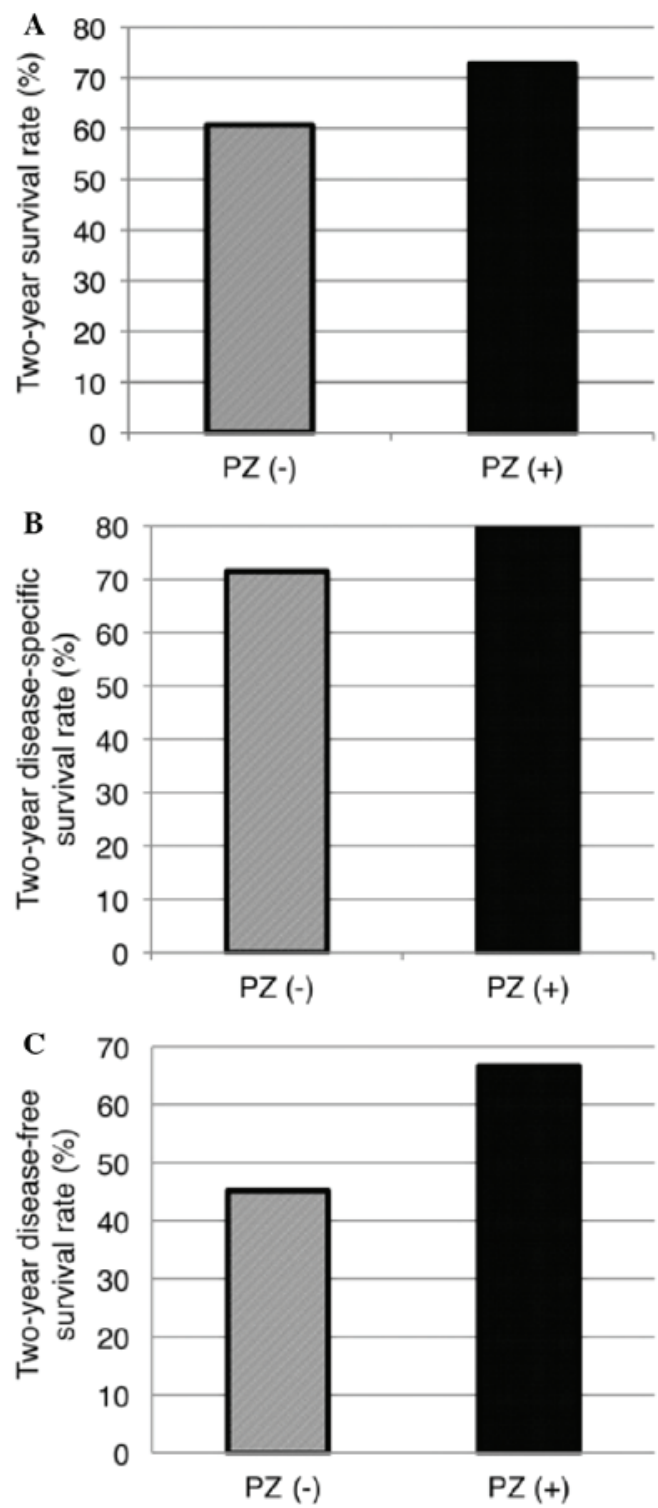

Figure 4. Survival outcomes at 2 years after the completion of treatment. (A) The overall survival rates were 72.7 and $60.7 \%$ in the PZ (+) and PZ (-) groups, respectively $(\mathrm{P}=0.55)$. (B) The disease-specific survival rates were 80.0 and $71.4 \%$ in the PZ (+) and PZ (-) groups, respectively $(\mathrm{P}=0.72)$. (C) The disease-free survival rates were 66.7 and $45.2 \%$ in the PZ (+) and PZ (-) groups, respectively $(\mathrm{P}=0.17)$. Patients who received the $\mathrm{PZ}$ oral rinse exhibited better survival outcomes. 
results in compromised oral food intake, thus leading to a requirement for enteral nutrition or intravenous hyperalimentation. Benzydamin has been reported to reduce the frequency of severe mucositis in head and neck cancer patients treated with lower doses of radiotherapy in a randomized controlled clinical trial (11). However, there are no established agents for the prevention of radiation-induced mucositis in patients with head and neck cancer receiving radical radiation therapy. We herein demonstrated that $\mathrm{PZ}$ oral rinse was highly tolerable and exhibited a good efficacy against chemoradiotherapy-induced oral mucositis in a higher number of head and neck cancer patients compared to previous reports $(9,10)$.

The survival outcomes of patients who receive PZ as supportive treatment are poorly understood (9). We were therefore concerned whether the survival outcome would be affected by $\mathrm{PZ}$, as it is known that radioscavangers may decrease the efficacy of chemoradiotherapy. Of note, the patients in the PZ (+) group exhibited better clinical outcomes compared to those in the PZ (-) group. Watanabe et al (9) reported that the oral food intake during radiotherapy was significantly improved with PZ treatment. Our data appear to be consistent with this previous report. In our study, the findings suggested that the use of PZ reduced the severity of mucositis induced by radiotherapy and improved the QOL, without negatively affecting the clinical outcomes. The improved QOL associated with PZ treatment was associated with improved patient health as a result of better nutrition. Therefore, the present study suggested that the use of PZ in patients with malignancies is acceptable.

It has been reported that PZ exerts its effects via a variety of cytoprotective mechanisms, including suppression of lipid peroxidation, reduction of the levels of various cytokines, inhibition of superoxide generation and promotion of the restoration of the gastric mucosa (4-7). In addition, PZ was recently reported to reduce radiation-induced damage and exert anti-inflammatory effects on the normal rectum of rats (8). Zinc supplementation has been reported to decrease the severity of radiation-induced mucositis in patients with head and neck cancer $(12,13)$. In addition, PZ, which is a chelate compound consisting of a zinc ion, L-carnosine, a $\beta$-alanine dipeptide and L-histidine, has been reported to be more effective in terms of superoxide scavenging activity compared to zinc sulfate (5). Therefore, the efficacy of PZ for radiation-induced mucositis appears to be promising.

It was recently reported that PZ reduced the severity of radiotherapy-induced mucositis in a randomized controlled clinical trial (9). However, the intake of PZ may have affected the results, since PZ may also act as a zinc supplement. PZ was not swallowed in the present study, in order to avoid the potential effects of PZ intake and to directly evaluate the effects of PZ on the oral mucosa. The PZ oral rinse was prepared with carboxyvinyl polymer as a base, in order to increase its stability and adherence to the mucosa (10). As a result, using the PZ oral rinse without swallowing exhibited good efficacy against radiation-induced mucositis and promoted mucosal healing in a retrospective analysis with a larger number of patients. Therefore, our data indicate that PZ exerts direct effects on the mucosa.

There were several limitations to the present study. We reported the outcomes following radiotherapy for head and neck cancer patients evaluated in a retrospective analysis. However, to the best of our knowledge, our study included the largest number of patients evaluated thus far and the treatment was performed at a single institution with high consistency. Although the follow-up period was insufficient to definitely evaluate survival outcome and late toxicity, we were able to confirm that the outcome was not inferior in patients who received the $\mathrm{PZ}$ oral rinse. Based on the present study, the $\mathrm{PZ}$ oral rinse may reduce the severity of acute mucositis caused by radiotherapy and significantly improve the patients' QOL. To fully elucidate the efficacy of the PZ oral rinse, future clinical trials with a longer follow-up period and a prospective randomized controlled study are required.

In conclusion, we performed a clinical trial comparing the effects of PZ on the incidence of severe oral mucositis and the symptoms induced by radiotherapy in patients with head and neck cancer. PZ was found to be highly effective in reducing the severity of mucositis and contributed to a better QOL for the patients, with no severe adverse effects and without compromising the tumor response to radiotherapy.

\section{Acknowledgements}

The authors would like to thank Drs Kota Kida, Takeshi Mohri, Kosuke Sagawa, Nobuhiro Uwa, Nobuo Saeki and Tomonori Terada from the Department of Otolaryngology, Hyogo College of Medicine (Nishinomiya, Hyogo, Japan) and Drs Kuniyasu Moridera and Kazuma Noguchi from the Department of Oral and Maxillofacial Surgery, Hyogo College of Medicine (Nishinomiya, Hyogo, Japan). We would also like to thank Ms. Sayaka Takahashi and Ms. Saori Kondo for their assistance with the data collection. This study was supported in part by unrestricted funding from Zeria Pharmaceutical Co., Ltd., (Tokyo, Japan).

\section{References}

1. National Comprehensive Cancer Network. NCCN Guidelines Head and Neck Cancers version 2, 2014.

2. Trotti A, Bellm LA, Epstein JB, et al: Mucositis incidence, severity and associated outcomes in patients with head and neck cancer receiving radiotherapy with or without chemotherapy: a systematic literature review. Radiother Oncol 66: 253-262, 2003.

3. Gu J, Zhu S, Li X, Wu H, Li Y and Hua F: Effect of amifostine in head and neck cancer patients treated with radiotherapy: a systematic review and meta-analysis based on randomized controlled trials. PLoS One 9: e95968, 2014.

4. Yoshikawa T, Naito Y, Tanigawa T, Yoneta T and Kondo M: The antioxidant properties of a novel zinc-carnosine chelate compound, N-(3-aminopropionyl)-L-histidinato zinc. Biochim Biophys Acta 1115: 15-22, 1991.

5. Yoshikawa T, Yamaguchi T, Yoshida N, et al: Effect of Z-103 on TNB-induced colitis in rats. Digestion 58: 464-468, 1997.

6. Omatsu T, Naito Y, Handa O, et al: Reactive oxygen species-quenching and anti-apoptotic effect of polaprezinc on indomethacin-induced small intestinal epithelial cell injury. J Gastroenterol 45: 692-702, 2010.

7. Opoka W, Adamek D, Plonka M, et al: Importance of luminal and mucosal zinc in the mechanism of experimental gastric ulcer healing. J Physiol Pharmacol 61: 581-591, 2010.

8. Doi H, Kamikonya N, Takada Y, et al: Efficacy of polaprezinc for acute radiation proctitis in a rat model. Int J Radiat Oncol Biol Phys 80: 877-884, 2011.

9. Watanabe T, Ishihara M, Matsuura K, Mizuta K and Itoh Y: Polaprezinc prevents oral mucositis associated with radiochemotherapy in patients with head and neck cancer. Int J Cancer 127: 1984-1990, 2010 
10. Nakayama M, Fujiwara M, Nakamura T, et al: Stability of polaprezinc-containing oral rinse and its clinical effectiveness against radiotherapy-induced oral mucositis. Iyakuhin Johogaku 15: $133-138,2013$.

11. Palazzi M, Tomatis S, Orlandi E, et al: Effects of treatment intensification on acute local toxicity during radiotherapy for head and neck cancer: prospective observational study validating CTCAE, version 3.0, scoring system. Int J Radiat Oncol Biol Phys 70: $330-337,2008$
12. Epstein JB, Silverman S, Paggiarino DA, et al: Benzydamine $\mathrm{HCl}$ for prophylaxis of radiation-induced oral mucositis: results from a multicenter, randomized, double-blind, placebo-controlled clinical trial. Cancer 92: 875-885, 2001.

13. Ertekin MV, Koc M, Karslioglu I and Sezen O: Zinc sulfate in the prevention of radiation-induced oropharyngeal mucositis: a prospective, placebo-controlled, randomized study. Int J Radiat Oncol Biol Phys 58: 167-174, 2004. 